\title{
A Rabbit Model for Sheep-Associated Malignant Catarrhal Fever Research: from Virus Infection to Pathogenesis Studies and Vaccine Development
}

\author{
Cristina W. Cunha ${ }^{1,2,3} \cdot$ Donal O'Toole $^{4} \cdot$ Naomi S. Taus ${ }^{1,3} \cdot$ Smriti Shringi $^{3} \cdot$ Donald P. Knowles $^{3} \cdot \mathrm{Hong} \mathrm{Li}^{1,3}$ \\ Published online: 17 July 2019 \\ (C) This is a U.S. Government work and not under copyright protection in the US; foreign copyright protection may apply 2019
}

\begin{abstract}
Purpose of Review Describe the implementation and use of rabbits as a laboratory model for sheep-associated malignant catarrhal fever (SA-MCF) research using cell-free ovine herpesvirus 2 (OvHV-2). Key considerations regarding the use of the model to generate consistent experimental data are presented and discussed in detail.

Recent Findings A major drawback to SA-MCF research is that OvHV-2 cannot be propagated in cell culture and experimental studies rely only on animals. An important research milestone was the finding that infectious cell-free OvHV-2 from nasal secretions of shedding sheep can be used to experimentally induce SA-MCF in various host species. Using this approach, we have demonstrated that rabbits can be infected with OvHV-2 and that infection parameters, such as dose-dependence, and clinical and pathological manifestations, resemble those in natural hosts.

Summary Rabbits are a reliable model for SA-MCF and represent an important resource for experimental research. The model is especially useful to investigate virus-host interactions and to evaluate MCF vaccine strategies.
\end{abstract}

Keywords Ovine herpesvirus $2(\mathrm{OvHV}-2) \cdot$ Malignant catarrhal fever (MCF) $\cdot$ Rabbits $\cdot$ Animal model

\section{Introduction}

Malignant catarrhal fever (MCF) is a severe and frequently fatal lymphoproliferative syndrome of multiple species in the order Artiodactyla, including species in the families Bovidae, Cervidae, Giraffidae, and Suidae. MCF is caused by one of several herpesviruses in the genus Macavirus, subfamily

This article is part of the Topical Collection on Virology

Cristina W. Cunha

cristina.cunha@usda.gov

1 Animal Disease Research Unit, USDA-Agricultural Research Service, Pullman, WA 99164-6630, USA

2 Paul G. Allen School for Global Animal Health, Washington State University, Pullman, WA 99164-7090, USA

3 Department of Veterinary Microbiology and Pathology, College of Veterinary Medicine, Washington State University, Pullman, WA 99164-7040, USA

4 Wyoming State Veterinary Laboratory, University of Wyoming, Laramie, WY 82070-6752, USA
Gammaherpesvirinae [1]. These viruses exist in nature as subclinical infections in species of the subfamilies Caprinae, Alcelaphinae, and Hippotraginae, which serve as their carrier hosts. Domestic sheep and wildebeest are the main reservoirs for ovine herpesvirus 2 (OvHV-2) and alcelaphine herpesvirus 1 (AlHV-1), respectively, the most studied MCF viruses.

In North America, MCF is caused predominantly by OvHV-2 and represents a serious threat to American bison due to their high susceptibility to disease. Significant economic losses caused by sheep-associated MCF (SA-MCF) have prompted research into effective strategies to control the disease and mitigate virus transmission.

Sheep-associated MCF is one of the few veterinary viral diseases for which its etiological agent, OvHV-2, has not been propagated in vitro. The absence of a culture system to propagate OvHV-2 hinders efforts to develop tools to study the virus and to reliably reproduce the disease experimentally. However, seminal work by our group in the early 2000s demonstrated that sheep naturally infected with OvHV-2 shed infectious virus through nasal secretions $[2 \bullet, 3 \bullet \bullet]$. Virus from this source created new opportunities to study SA-MCF and, since then, experimental disease has been consistently induced in several clinically susceptible species, including domestic 
cattle, American bison, pigs, and rabbits [4, 5, 6•, 7]. Based on these experiments, we were able to demonstrate that susceptibility to SA-MCF varies appreciably among species. For instance, American bison are highly susceptible to the disease, whereas cattle are comparatively more resistant $[5,8]$.

Although natural hosts are generally the best experimental animal models for research, there are limitations on their use in SA-MCF experimental studies. Bison are difficult to handle, require special facilities, and are expensive to maintain. Routine experimental use of cattle is less problematic but because of their relative resistance to infection, reliable induction of clinical disease requires high infectious doses of OvHV-2, which is difficult to obtain. In this context, the use of a small animal laboratory model that is both easy to maintain and susceptible to SA-MCF is beneficial for experimental research. Laboratory rabbits fit these criteria. While we are not aware of MCF in free-ranging populations of rabbits, the disease can be reliably induced experimentally in laboratory rabbits using relatively low doses of OvHV-2 $[6 \bullet, 9]$. Importantly, initial infection, virus dissemination, disease manifestations, and host immune responses following experimental challenge with OvHV-2 in rabbits are similar to those in bison $[10,11 \bullet \bullet]$. After performing OvHV-2 experimental infections in rabbits for several studies, we have now established that rabbits are a reliable and valid model for SA-MCF and in this article, we review specific aspects of their use to advance MCF research.

\section{History of the Use of Rabbits in MCF Research}

Rabbits (Oryctolagus cuniculus) are small mammals in the family Leporidae of the order Lagomorpha. They are commonly used as experimental models in many research fields as they are docile, easy to house, handle, and observe. Laboratory rabbits are widely available and cost-effective for research compared to larger animal models. Additionally, rabbit-specific reagents, particularly to assess immunological responses, are commercially available.

Initial reports of experimental use of rabbits in MCF research come from the 1920s and 30s [12]. From that time until the 1970s, laboratory rabbits were used for AlHV-1transmission studies using infectious materials from cattle with wildebeest-associated MCF [13]. The majority of these studies focused on virus-host interactions, including targeted cells, lymphoid proliferation, and host immune responses [14-19]. In the early $1980 \mathrm{~s}$, scientists at the Moredun Research Institute in Scotland induced MCF in rabbits using tissue suspensions and leukocytes from a clinically infected red deer (Cervus elaphus) that had been exposed to sheep and subsequently described the clinical signs and lesions in rabbits with OvHV-2-induced MCF [20, 21]. An associated milestone from their work was the establishment of an OvHV-2-infected lymphoblastoid cell line from a rabbit with SA-MCF [22].
Although the virus does not seem to replicate in these cells and viral particles are not detected, it could induce MCF. Over the last three decades, many studies conducted in rabbits used intravenous inoculation of either OvHV-2-infected lymphoblastoid cell lines or virulent cell-associated strains of AlHV-1 (e.g., C500 strain) to investigate the pathogenesis of MCF, with particular emphasis on the nature of lymphoproliferation [23-25]. More recently, cell-free OvHV-2 obtained from sheep nasal secretions led to a reliable source of virus for in vivo studies [2•]. Experimental challenges using intranasal nebulization of sheep nasal secretions containing infectious OvHV-2 have been used extensively to infect various host species, including rabbits $[3 \bullet \bullet, 4,5,6 \bullet, 7,10,11 \bullet, 26]$. These experiments resulted in much of what is currently known about OvHV-2 infection and disease development in several natural hosts and validated rabbits as a suitable animal model for SA-MCF research.

\section{Induction of SA-MCF in Rabbits Using Cell-Free OvHV-2}

Rabbits can be infected with OvHV-2 following experimental inoculation of cell-associated virus derived from infected animals or lymphoblastoid cells [12, 20, 25, 27]. A disadvantage of this approach is that infection and induction of disease are inconsistent, as it is difficult to standardize inocula needed to replicate challenge studies. Cell-free infectious OvHV-2 present in nasal secretions of shedding sheep offer a more reliable source of infectious virus since these can be stored, quantified, and standardized for in vivo experiments [3••].

Below, we describe specific aspects of the cell-free OvHV2 preparation and experimental procedures that are followed in our laboratory to study SA-MCF using the rabbit model.

\section{Generation of Standardized Cell-Free OvHV-2 Stocks} Preparation of cell-free OvHV-2 stocks for animal experiments relies on the collection of nasal secretions from infected sheep during intense viral shedding episodes. Although OvHV-2 can be continuously detected in sheep nasal secretions, shedding of high levels of virus is unpredictable and occurs as brief episodes that usually lasts less than $24 \mathrm{~h}[2 \bullet$, 28]. This makes the identification of a high-shedding sheep a laborious process. To obtain infectious virus, OvHV-2infected adolescent sheep (6-9-month-old), which are more prone to shed the virus, must be monitored daily for intense viral shedding episodes, i.e., presence of high concentrations of viral DNA $\left(10^{5}-10^{8}\right.$ OvHV-2 genome copies per microgram of total DNA) in nasal secretions $[28,29]$. This involves collecting nasal secretions using a cotton-tipped swab and immediately testing them for the quantity of OvHV-2 DNA using an OvHV-2 specific quantitative PCR [30]. Once a high concentration of OvHV-2 DNA is detected in a sheep, 
additional nasal secretions ( 4 to 8 swabs) are collected from that animal within a few hours of the first swab to obtain a larger sample. The process continues until several highshedding sheep are identified and collected from, generating multiple nasal secretion samples containing high concentrations of OvHV-2. In our experience, about 15 sheep need to be monitored over 3 months to identify 20 to 25 high shedding episodes. Upon collection, each nasal swab is placed in cold phosphate buffer solution (PBS) containing 5\% chicken egg albumin (approximately $1 \mathrm{ml}$ of PBS per swab), clarified by low speed centrifugation, aliquoted, and stored in liquid nitrogen. To generate standard OvHV-2 stocks, representative aliquots from each individual sheep nasal secretions are pooled, quantified, and re-aliquoted for future use. In our laboratory, OvHV-2 stocks are stored in liquid nitrogen as aliquots containing $10^{8} \mathrm{OvHV}-2$ genome copies per milliliter.

Infectivity of viral stocks is tested by inoculating groups of naïve susceptible hosts with secretions serially diluted based on OvHV-2 DNA copy numbers. Following inoculation, seroconversion, detection of viral DNA in the blood, and/or clinical signs are monitored, with the goal of identifying the minimum dose capable of establishing infection and/or disease. This approach circumvents the absence of a culture system to propagate and test OvHV-2 infectivity in vitro and allows standardization of inocula based on viral genome copies. In our laboratory, we have used mostly sheep to test the infectivity of new viral stock preparations and considered seroconversion and detection of viral DNA in the blood as indicators of infection. Because sheep can be infected with lower doses of virus ( $10^{3}$ OvHV-2 genome copies) as compared with other animal species, their use in this type of experiment helps to conserve the stock $[3 \bullet \bullet, 31]$. A disadvantage of this approach is that it necessitates access to OvHV-2-negative sheep, which may be challenging to find as the vast majority of adult sheep under standard husbandry conditions are infected with the virus $[32,33]$. Alternatively, rabbits can be used to test OvHV-2 stocks for infectivity by using detection of viral DNA and disease development as indicators of infection. However, an inoculum containing at least $10^{5}$ OvHV-2 genome copies is needed to reliably infect and induce disease in rabbits [9, 31].

Intranasal Nebulization Cell-free OvHV-2 obtained from sheep nasal secretions infects and induces disease in susceptible hosts when inoculated by respiratory aerosol, mimicking natural transmission. Such inocula fail to induce infection or disease when introduced intravenously or intraperitoneally $[6 \bullet, 7,10,26]$. Although the reason for this is unknown, it is probably related to changes in virus components and/or cell tropism switching during initial infection and subsequent dissemination, as occurs with some other herpesviruses [34, 35].

Here, we offer a detailed protocol for inoculating rabbits with standardized cell-free OvHV-2 preparations using intranasal nebulization. First, the virus stock is diluted in a PBS to a total volume of $2 \mathrm{ml}$ containing the desired challenge dose. The inoculum is loaded into a nebulizer connected to a plastic cone covering the animal's nose and aerosolized for 6 min. During nebulization, proper handling of rabbits and accurate timing for each procedure significantly reduce variation in the inoculum dose, which is critical to ensure consistent experimental OvHV-2 infections. It is important to use the same viral stock in any series of experiments within a project. To help avoid unrelated infections, the use of specific pathogen-free rabbits and administration of prophylactic antibiotic therapy (e.g., enrofloxacin, $5 \mathrm{mg} / \mathrm{kg}$; three intramuscular doses at $24 \mathrm{~h}$ interval) concurrent to OvHV-2-infected or mock nasal secretion inoculations is advised.

Defining OvHV-2 Infection Parameters in Rabbits Although SA-MCF can be consistently induced in rabbits using intranasal delivery of cell-free OvHV-2, infection parameters need to be defined in a case-to-case manner considering both the source of rabbits and the virus stock.

In our initial attempts to induce SA-MCF, outcomes differed depending on the rabbits' origin. New Zealand white rabbits from two suppliers were inoculated by intranasal nebulization with the same dose of OvHV-2 (10 ${ }^{7}$ viral genome copies) from a single pool of standardized viral stock. All rabbits had similar body weights and were inoculated at 10 weeks of age $[6 \bullet, 36]$. In study \#1, only 5 out of 8 rabbits $(62 \%)$ were infected with OvHV-2 and developed SA-MCF. By contrast, all 6 rabbits $(100 \%)$ in study \#2 developed disease. Although susceptibility to OvHV-2 infection was not the same for each rabbit group, the time for development of disease was similar, with incubation periods averaging $30 \pm$ 4.3 days post-inoculation (DPI) and $27 \pm 1.6 \mathrm{DPI}$ in studies $\# 1$ and \#2, respectively (Fig. 1a). Since there was no difference in age, weight, or dose of inoculum in these two studies, the most likely explanation for the difference in susceptibility to OvHV-2 infection was the origin of the animals. Information obtained from the two rabbit suppliers revealed that distinct breeding programs were followed by each vendor. The company that supplied the rabbits for study \#1 had a strictly outbred program, while the company that supplied rabbits for study \#2 used a highly inbred program. Although the reason for the differences in OvHV-2 susceptibility is not completely understood, the results suggest that rabbits from different sources may show distinct infection rates, most likely reflecting genetic variations and degree of inbreeding among rabbits. Thus, pilot experiments to define the OvHV-2 inoculum dose necessary to induce SA-MCF in a given population of rabbits using a defined virus preparation are advisable to implement the model in different laboratory settings. Once these parameters are established, the model is robust and consistent among experiments. 

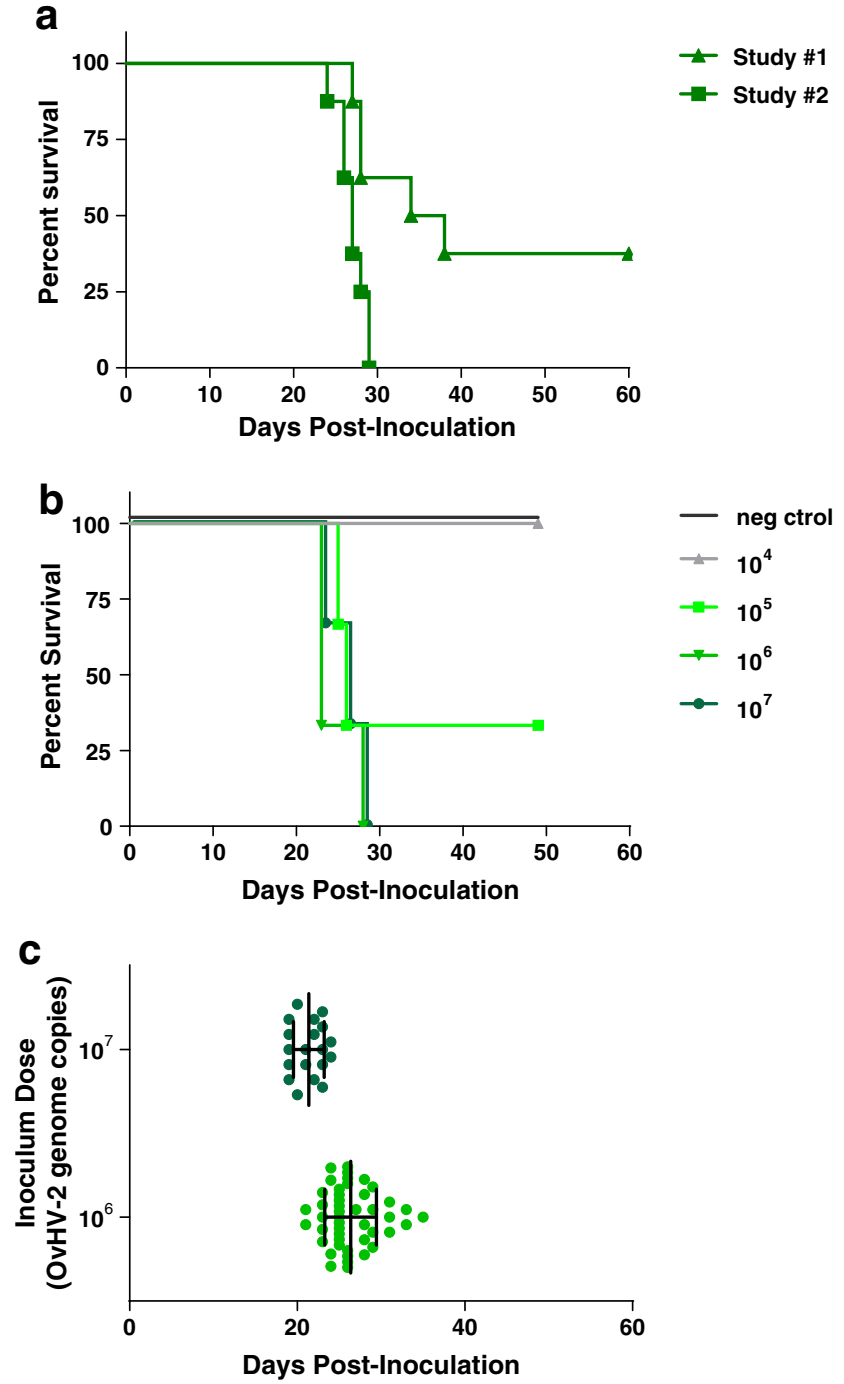

Fig. 1 Time for development of SA-MCF in rabbits inoculated with cellfree OvHV-2 by intranasal nebulization at 10 weeks of age. a Studies performed with rabbits from different sources; all animals were inoculated with $10^{7}$ OvHV-2 genome copies. b Rabbits inoculated with serial doses of OvHV-2 or a mock inoculum (negative control). $\mathbf{c}$ Rabbits inoculated with different doses of OvHV-2 from the same viral stock over multiple experiments in our laboratory; bars indicate mean and standard deviation

The first study to evaluate OvHV-2 infectivity in rabbits in our laboratory using various viral doses inoculated by intranasal nebulization was performed using 10 -week-old New Zealand rabbits obtained from the vendor (inbred program) used in study \#2 described above [9]. Four groups of rabbits $(n=3)$ were nebulized with sheep nasal secretions containing $10^{7}, 10^{6}, 10^{5}$, or $10^{4}$ OvHV-2 genome copies. One group of rabbits received nasal secretions obtained from an OvHV-2 uninfected sheep (negative control group). As illustrated in Fig. $1 \mathrm{~b}$, inocula containing $10^{7}$ and $10^{6}$ OvHV-2 genome copies resulted in $100 \%$ infection and mortality due to MCF, while $67 \%$ mortality ( 2 of 3 rabbits) was observed in rabbits receiving a dose of $10^{5}$ copies. Regardless of the inoculum dose, all rabbits with clinical signs of MCF were euthanized between 23 and 28 DPI. The rabbit that survived challenge with a $10^{5}$ copies inoculum was infected with OvHV-2, as demonstrated by PCR and serology, but did not develop disease until 60 DPI, when the experiment was terminated. No rabbit in the group inoculated with $10^{4}$ copies or in the control group was infected during the 60-day post-inoculation period. The study indicated that an inoculum of at least $10^{5}$ OvHV-2 genome copies of virus stock is necessary to infect rabbits and that reliable induction of disease requires higher doses of virus (viz. $10^{6}$ or $10^{7}$ copies). These results confirmed that development of MCF in rabbits is dose-dependent, as was previously demonstrated in bison, cattle, and pigs $[5,7,8]$.

The results of the above study served as a guide for future experiments performed in our laboratory. Since then, additional 10-week-old, inbred New Zealand rabbits have been intranasally inoculated with $10^{6}$ or $10^{7}$ OvHV-2 genome copies from the same viral stock in multiple studies. Infection and development of MCF were confirmed in 46 of 48 (96\%) rabbits inoculated with $10^{6}$ OvHV-2 genome copies while all $(100 \%, n=19)$ animals inoculated with $10^{7} \mathrm{OvHV}-2$ genome copies developed disease. Collectively, these experiments allowed evaluation of the course of infection, such as the first detection of OvHV-2 DNA in blood and the development of clinical disease, following challenge with different inoculum doses. Viral DNA in PBL was detectable in blood as soon as 14 DPI, regardless the initial dose of virus. However, rabbits inoculated with $10^{7}$ OvHV-2 DNA copies tended to develop MCF earlier (21.4 \pm 1.8 DPI) than ones receiving $10^{6}(26.4 \pm$ 3.1 DPI). Figure 1c illustrates incubation period data obtained from all the experiments combined. Although the nature of the data collected from various studies does not allow for statistical analysis, more variation and longer incubation periods were observed in animals inoculated with the lower inocula. Depending on the purpose of the experiment, and notwithstanding the absence of $100 \%$ infection rates, experiments using $10^{6}$ OvHV-2 genome copies may be used, since it conserves viral stocks. However, this may necessitate more animals and somewhat longer studies to allow for statistical analysis.

By performing the experiments described above, we were able to define infection parameters to reliably induce MCF in rabbits using the virus stock available in our laboratory. Even though the results cannot be directly extrapolated to other research groups, they serve as a starting point and a guide for the establishment of the model in different laboratories.

\section{SA-MCF in Rabbits}

Induction of MCF in rabbits by intranasal inoculation with OvHV-2 derived from sheep nasal secretions was first reported by our group in 2008 , followed by more detailed reports in 
2011 and $2013[6 \cdot, 11 \bullet \cdot, 36]$. Overall, an intranasal challenge containing $10^{6}-10^{7}$ viral DNA copies of standardized cell-free OvHV-2 causes clinical disease in rabbits. Following inoculation, rabbits should be checked at least once daily for development of clinical signs, such as fever $\left(>40^{\circ} \mathrm{C}\right)$, which usually occurs $2-3$ weeks post-inoculation. Thereafter, they should be examined more frequently (3-4 times a day), since some animals develop severe clinical signs more quickly than others. In our laboratory, experimental animals are euthanized within $48 \mathrm{~h}$ of the onset of fever, or immediately if additional clinical signs, such as depression, anorexia, adipsia, diarrhea, oculonasal discharge, and neurological signs, are present. Immediately after euthanasia, necropsy is performed and selected organs collected for further analysis.

In rabbits with SA-MCF, the lesion pattern is consistent and largely mirrors that in susceptible artiodactyls. These comprise lymphoid hyperplasia, vasculitis-perivasculitis, and interstitial lymphocytic inflammation in multiple organs with the predominance of large lymphocytes $[6 \cdot 23$, 36]. The most consistent gross lesions in OvHV-2-infected rabbits following aerosolization of cell-free virus are observed in the liver and to a lesser extent in the spleen, lymph nodes, and lymph node aggregates [36]. Hepatic lesions consist of disseminated pinpoint tan foci due to diffuse portal-periportal hepatitis and hepatocellular necrosis, with bridging lesions between adjacent portal areas [6•]. Cholangitis is a consistent feature, with hyperplasia and epithelial necrosis/apoptosis in biliary epithelium and with some degree of portal phlebitis-arteriolitis. Large areas of hepatocellular necrosis are associated with granulomatous inflammation, including the presence of giant cells (polykaryons). Arteriolitis-phlebitis also occurs in the bronchial circulation, concurrent with interstitial pneumonia in peribronchiolar parenchyma. Lymphoid hyperplasia involves the T cell-rich paracortex of lymph nodes and gutassociated lymphoid tissues. With the exception of hyperplastic gut-associated lymphoid aggregates in cecum, gastrointestinal lesions are mild or absent. Small, widely scattered ulcers may be present in the cecum and the tongue. Encephalitis and meningitis are absent in many terminally affected rabbits. Changes in cornea and conjunctiva can be surprisingly mild, overt clinical keratoconjunctivitis notwithstanding. Apart from above, lesions found in rabbits with SA-MCF but absent in other species are necrotizing granulomatous hepatitis and lymphadenitis [37]. In some rabbits with SA-MCF, pneumonia has also granulomatous features, specifically the presence of giant cells. The basis for this granulomatous response in rabbits is unclear. Although gross and histologic lesions in OvHV-2- and AlHV-1-induced MCF in rabbits are qualitatively similar, necrosis observed in the liver, mesenteric lymph nodes, and appendix is characteristic of rabbits infected with OvHV-2 but not present in AlHV-1-infected animals [20, 23, 37].
Consistent with these morphological changes, OvHV-2 DNA can be detected in both lymphoid and non-lymphoid tissues. The liver, mesenteric lymph nodes, and lungs tend to have higher viral loads, with an average of $10^{7}-10^{8}$ OvHV-2 genome copies per microgram of total tissue DNA, as compared to other organs $[6 \bullet, 11 \bullet \bullet$. Furthermore, OvHV-2 nucleic acids co-localized with lesions have been recently demonstrated using in situ hybridization $[6 \bullet, 11 \bullet, 38]$.

In spite of advances in the last few decades regarding experimental induction of MCF using either OvHV-2 or AlHV-1 in natural and experimental hosts, the pathogenesis of the disease is still poorly understood and a matter of debate. In bison and rabbits, there is an initial round of viral replication restricted to the lungs following OvHV-2 cell-free intranasal inoculation $[10,11 \bullet \cdot]$. Initial replication limited to pulmonary tissue suggests that replication in the lungs is required to establish infection and generate progeny viruses that subsequently infect lymphocytes and disseminate systemically. The first SA-MCF pathogenesis study in rabbits was reported by Buxton and collaborators in 1984. These authors proposed that disease was due to dysfunction of immunoregulatory cells, resulting in benign polyclonal $\mathrm{T}$ lymphocyte proliferation [21]. More recent studies have not only confirmed and expanded some of the previous finds but also, by comparing OvHV-2 and AlHV-1-infected rabbits, have identified some differences in the pathogenesis of the disease caused by these two viruses [23, 24, 36, 39, 40]. For instance, in AlHV-1infected animals, extensive proliferation of $\mathrm{CD} 8^{+} \mathrm{T}$ cells expressing a cytotoxic phenotype in both lymphoid and nonlymphoid tissues has been demonstrated [23,39]. By contrast, accumulation of cytotoxic and regulatory cells, consisting of $\mathrm{T}$ cells, B cells, and monocytes, is detected in SA-MCF-affected animals $[23,36,40]$. More importantly, in rabbits with AlHV1-induced MCF, a latent infection is present in tissues, as demonstrated by the absence of detectable viral transcripts that are characteristic of productive infections [40]. The same research group provided convincing evidence for the importance of the latency-associated nuclear antigen in the development of disease, resulting in the proposal that latent infection is an essential aspect in the pathogenesis of AlHV-1-induced MCF [41]. By contrast, gene expression indicative of both latent and productive cycles has been demonstrated in cultured T cells from OvHV-2-infected rabbits [42, 43]. In addition, the concentration of ORF 25 transcripts, a marker for virus replication, was positively correlated with severity of lesions in rabbits and bison with SA-MCF $[10,11 \bullet \cdot]$. Collectively, these data suggest that a mixture of latent and replicating virus, leading to immune cytotoxicity and dysregulation, may be involved in the pathogenesis of SA-MCF [10, 44]. Additional studies are necessary to clarify mechanisms leading to cell activation and immune dysregulation following OvHV-2 and AlHV-1 infection, as well as the role played by the viruses or their components in the development of MCF. 
In this scenario, given the similarities of the disease in rabbits and in natural hosts, the rabbit model will continue to be an important tool to dissect the pathogenesis of the disease caused by both OvHV-2 and AlHV-1.

\section{MCF Vaccine Development}

One major goal of MCF research is the development of effective vaccines to protect clinically susceptible animals from natural disease caused by MCF viruses. It has been previously demonstrated that AlHV-1-neutralizing antibodies, induced by vaccination with an attenuated AlHV-1, can protect animals from lethal AlHV-1 challenge, confirming that achieving protection from MCF using vaccination is an accomplishable goal [45].

The absence of an in vitro system to propagate OvHV-2 not only makes an attenuated vaccine for this virus unlikely but also makes detection of neutralizing antibodies and identification of viral targets a challenging task. Therefore, alternative approaches for vaccine development and monitoring protective immune responses are necessary. The possibility of using rabbits to test new vaccine targets and to assess neutralizing antibodies proffers opportunities to advance research using a reliable animal model.

The rabbit model has been effectively used to evaluate OvHV-2 glycoproteins capable of inducing neutralizing antibody responses [46, 47]. In one of these studies, rabbits were immunized with recombinant DNA plasmids expressing OvHV-2 glycoprotein $\mathrm{B}(\mathrm{gB}), \mathrm{gH}$, and $\mathrm{gL}$ delivered into the skin by a biolistic method, resulting in successful production of hyperimmune sera. The sera were then used to treat cellfree OvHV-2 prior to intranasal inoculation into rabbits. Animals infected with antibody-treated virus did not develop disease, confirming that anti-OvHV-2 gB, gH, and gL antibodies can neutralize OvHV-2 [46]. Induction of anti-OvHV-2 antibodies in rabbits has also been demonstrated using an AlHV-1 chimeric virus that had its gB-encoding gene replaced by the OvHV-2 homolog gene [47]. Testing this AlHV-1 mutant virus in challenge experiments in the rabbit model will allow for its evaluation as a potential vaccine for both AlHV-1 and OvHV-2.

Although the target animals for any SA-MCF vaccine are large ruminants, particularly bison and cattle, preliminary experiments in a laboratory animal model are helpful to assess new vaccine constructs for induction of protective immune responses and to guide more complex and expensive trials in large animals. An important feature in favor of using rabbits for these purposes is that the expression pattern of immune genes associated with both cellular and humoral responses following OvHV-2 infection in rabbits and bison is very similar $[10,11 \bullet \cdot]$. Therefore, it is expected that data obtained in rabbits regarding OvHV-2 vaccine candidates, delivery systems, adjuvants, and host responses will be critical in identifying vaccine strategies and protocols that warrant further investigation in one or more susceptible ungulate species.

\section{Conclusion}

With the lack of an in vitro system to propagate OvHV-2, SAMCF research relies heavily on animal experiments. Since routine use of natural hosts is expensive and logistically impractical, a reliable laboratory animal model is critical for research progress. Rabbits are susceptible to OvHV-2 infection, and a disease similar to that in natural hosts can be reliably induced following initial experimental system optimization. Therefore, rabbits will continue to be used for the foreseeable future to better understand the pathogenesis of SAMCF and for the development of MCF vaccines. By describing steps carried out in our laboratory to implement a rabbit model for SA-MCF studies using cell-free OvHV-2, this review aims to highlight important aspects to consider in order to achieve consistent and reliable experimental data when using rabbits for OvHV-2 research.

Acknowledgments We thank the many colleagues, staff and students, past and present, involved in the experiments performed in our laboratory and described in this review. We also thank Dr. Reginaldo Bastos for the critical review of the manuscript.

Funding Preparation of this article and the experiments performed by the authors were supported by USDA-RS-CWU grant 2090-32000-037-00D.

\section{Compliance with Ethical Standards}

Conflict of Interest Cristina W. Cunha, Donal O'Toole, Naomi S. Taus, Smriti Shringi, Donald P. Knowles, and Hong Li each declare no potential conflicts of interest.

\section{References}

Papers of particular interest, published recently, have been highlighted as:

- Of importance

- Of major importance

1. Davison AJ. Herpesvirus systematics. Vet Microbiol. 2010;143:5269.

2. Li H, Taus NS, Lewis GS, Kim O, Traul DL, Crawford TB. Shedding of ovine herpesvirus 2 in sheep nasal secretions: the predominant mode for transmission. J Clin Microbiol. 2004;42: 5558-64 This was the first study describing the pattern of OvHV-2 shedding in sheep, which allowed nasal secretions to be collected as a source of virus for experimental studies.

3.• Taus NS, Traul DL, Oaks JL, Crawford TB, Lewis GS, Li H. Experimental infection of sheep with ovine herpesvirus 2 via aerosolization of nasal secretions. J Gen Virol. 2005;86:575-9 The 
study demonstrated that nasal secretions from OvHV-2 shedding sheep contain infectious virus capable of infecting naïve sheep when inoculated experimentally by intranasal nebulization.

4. O'Toole D, Taus NS, Montgomery DL, Oaks JL, Crawford TB, Li H. Intra-nasal inoculation of American bison (Bison bison) with ovine herpesvirus-2 (OvHV-2) reliably reproduces malignant catarrhal fever. Vet Pathol. 2007;44:655-62.

5. Taus NS, Oaks JL, Gailbreath K, Traul DL, O'Toole D, Li H. Experimental aerosol infection of cattle (Bos taurus) with ovine herpesvirus 2 using nasal secretions from infected sheep. Vet Microbiol. 2006;116:29-36.

6. Gailbreath KL, Taus NS, Cunha CW, Knowles DP, Li H. Experimental infection of rabbits with ovine herpesvirus 2 from sheep nasal secretions. Vet Microbiol. 2008;132:65-73 The article report that rabbits can be experimentally infected with OvHV2 by intranasal nebulization of virus obtained from sheep nasal secretions. Detailed description of SA-MCF lesions in rabbits compared with cattle and bison is also presented.

7. Li H, Brooking A, Cunha CW, Highland MA, O'Toole D, Knowles DP, et al. Experimental induction of malignant catarrhal fever in pigs with ovine herpesvirus 2 by intranasal nebulization. Vet Microbiol. 2012;159:485-9.

8. Gailbreath KL, O'Toole D, Taus NS, Knowles DP, Oaks JL, Li H. Experimental nebulization of American bison (Bison bison) with low doses of ovine herpesvirus 2 from sheep nasal secretions. Vet Microbiol. 2010;143:389-93.

9. Li H, Cunha CW, O'Toole D, Nicola AV, Knowles DP, Taus NS. Development of an in vivo system to measure antibody-blocking of ovine herpesvirus 2 entry. J Virol Methods. 2013;188:104-7.

10. Cunha CW, Gailbreath KL, O'Toole D, Knowles DP, Schneider DA, White SN, et al. Ovine herpesvirus 2 infection in American bison: virus and host dynamics in the development of sheepassociated malignant catarrhal fever. Vet Microbiol. 2012;159: 307-19.

11.• Cunha CW, O'Toole D, Taus NS, Knowles DP, Li H. Are rabbits a suitable model to study sheep-associated malignant catarrhal fever in susceptible hosts? Vet Microbiol. 2013;163:358-63 The study confirms that following intranasal inoculation of cell-free OvHV-2 in rabbits, initial infection, virus dissemination, disease manifestations, and host immune responses resemble those in bison, indicating that the rabbits can be used as a model for SAMCF studies.

12. Daubney R, Hudson JR. Transmission experiments with bovine malignant catarrh. J Comp Pathol. 1936;49:63-89.

13. Plowright $\mathrm{W}$. Malignant catarrhal fever virus. In: Dinter $Z$, Morein B, editors. Virus infections of ruminants. New York: Elsevier Science Publishers B.V; 1990. p. 123-50.

14. Edington N, Plowright $\mathrm{W}$. The protection of rabbits against the herpesvirus of malignant catarrhal fever by inactivated vaccines. Res Vet Sci. 1980;28:384-6.

15. Mushi EZ. The proliferation of malignant catarrhal fever virus in cattle and rabbits. Bull Anim Health Prod Africa. 1980;28:85-9.

16. Patel JR, Edington N. The detection of the herpesvirus of bovine malignant catarrhal fever in rabbit lymphocytes in vivo and in vitro. J Gen Virol. 1980;48:437-44.

17. Rossiter PB, Mushi EZ, Plowright W. Antibody response in cattle and rabbits to early antigens of malignant catarrhal fever virus in cultured cells. Res Vet Sci. 1978;25:207-10.

18. Rossiter PB. Proliferation of T lymphoblasts in rabbits fatally infected with the herpes virus of malignant catarrhal fever. Clin Exp Immunol. 1983;54:547-53.

19. Rurangirwa FR, Mushi EZ. Target cells for malignant catarrhal fever virus in rabbits. Vet Res Commun. 1982;5:285-8.

20. Buxton D, Reid HW. Transmission of malignant catarrhal fever to rabbits. Vet Rec. 1980;106:243-5.
21. Buxton D, Reid HW, Finlayson J, Pow I. Pathogenesis of 'sheepassociated' malignant catarrhal fever in rabbits. Res Vet Sci. 1984;36:205-11.

22. Reid HW, Buxton D, Pow I, Finlayson J, Berrie EL. A cytotoxic Tlymphocyte line propagated from a rabbit infected with sheep associated malignant catarrhal fever. Res Vet Sci. 1983;34:109-13.

23. Anderson IE, Buxton D, Campbell I, Russell G, Davis WC, Hamilton MJ, et al. Immunohistochemical study of experimental malignant catarrhal fever in rabbits. J Comp Pathol. 2007;136:15666.

24. Schock A, Reid HW. Characterisation of the lymphoproliferation in rabbits experimentally affected with malignant catarrhal fever. Vet Microbiol. 1996;53:111-9.

25. Swa S, Wright H, Thomson J, Reid H, Haig D. Constitutive activation of Lck and Fyn tyrosine kinases in large granular lymphocytes infected with the gamma-herpesvirus agents of malignant catarrhal fever. Immunology. 2001;102:44-52.

26. Li H, O'Toole D, Kim O, Oaks JL, Crawford TB. Malignant catarrhal fever-like disease in sheep after intranasal inoculation with ovine herpesvirus-2. J Vet Diagn Investig. 2005;17:171-5.

27. Reid HW, Buxton D, Pow I, Finlayson J. Malignant catarrhal fever: experimental transmission of the 'sheep-associated' form of the disease from cattle and deer to cattle, deer, rabbits and hamsters. Res Vet Sci. 1986;41:76-81.

28. Li H, Hua Y, Snowder G, Crawford TB. Levels of ovine herpesvirus 2 DNA in nasal secretions and blood of sheep: implications for transmission. Vet Microbiol. 2001;79:301-10.

29. Cunha CW, Traul DL, Taus NS, Oaks JL, O'Toole D, Davitt CM, et al. Detection of ovine herpesvirus 2 major capsid gene transcripts as an indicator of virus replication in shedding sheep and clinically affected animals. Virus Res. 2008;132:69-75.

30. Traul DL, Taus NS, Oaks JL, O'Toole D, Rurangirwa FR, Baszler TV, et al. Validation of non-nested and real-time PCR for diagnosis of sheep-associated malignant catarrahal fever in clinical samples. J Vet Diagn Investig. 2007;19:405-8.

31. Li H, Cunha CW, Taus NS, Knowles DP. Malignant catarrhal fever: inching toward understanding. Annu Rev Anim Biosci. 2014;2: 209-33.

32. Li H, Shen DT, Jessup DA, Knowles DP, Gorham JR, Thorne T, et al. Prevalence of antibody to malignant catarrhal fever virus in wild and domestic ruminants by competitive-inhibition ELISA. J Wildl Dis. 1996;32:437-43.

33. Zarnke RHLH, Crawford TB. Serum antibody prevalence of malignant catarrhal fever viruses in seven wildlife species from Alaska. J Wildl Dis. 2002;38:500-4.

34. Nguyen CC, Kamil JP. Pathogen at the gates: human cytomegalovirus entry and cell tropism. Viruses. 2018;10:704.

35. Shannon-Lowe C, Rowe M. Epstein Barr virus entry; kissing and conjugation. Curr Opin Virol. 2014;4:78-84.

36. Li H, Cunha CW, Gailbreath KL, O'Toole D, White SN, Vanderplasschen A, et al. Characterization of ovine herpesvirus 2-induced malignant catarrhal fever in rabbits. Vet Microbiol. 2011;150:270-7.

37. O'Toole $\mathrm{D}, \mathrm{Li} \mathrm{H}$. The pathology of malignant catarrhal fever, with an emphasis on ovine herpesvirus 2. Vet Pathol. 2014;51:437-52.

38. Pesavento PA, Cunha CW, Li H, Jackson K, O'Toole D. In situ hybridization for localization of ovine herpesvirus 2 , the agent of sheep-associated malignant catarrhal fever, in formalin-fixed tissues. Vet Pathol. 2018;56:78-86.

39. Dewals B, Boudry C, Farnir F, Drion PV, Vanderplasschen A. Malignant catarrhal fever induced by alcelaphine herpesvirus 1 is associated with proliferation of CD8 T cells supporting a latent infection. PLoS One. 2008;3:e1627.

40. Dewals B, Vanderplasschen A. Malignant catarrhal fever induced by alcelaphine herpesvirus 1 is characterized by an expansion of activated $\mathrm{CD} 3+\mathrm{CD} 8+\mathrm{CD} 4-\mathrm{T}$ cells expressing a cytotoxic 
phenotype in both lymphoid and non-lymphoid tissues. Vet Res. 2011;42:95.

41. Palmeira L, Oa S, Van Campe W, Boudry C, Roels S, Myster F, et al. An essential role for $\mathrm{g}$-herpesvirus latency-associated nuclear antigen homolog in an acute lymphoproliferative disease of cattle. PNAS. 2013;110:E1933-42.

42. Rosbottom J, Dalziel RG, Reid HW, Stewart JP. Ovine herpesvirus 2 lytic cycle replication and capsid production. J Gen Virol. 2002;83:2999-3002.

43. Thonur L, Russell GC, Stewart JP, Haig DM. Differential transcription of ovine herpesvirus 2 genes in lymphocytes from reservoir and susceptible species. Virus Genes. 2006;32:27-35.

44. Nelson DD, Davis WC, Brown WC, Li H, O'Toole D, Oaks JL. CD8+/perforin+/WC1 - [gamma][delta] T cells, not CD8+ [alpha][beta] T cells, infiltrate vasculitis lesions of American bison (Bison bison) with experimental sheep-associated malignant catarrhal fever. Vet Immunol Immunopathol. 2010;136:284-91.
45. Haig DM, Grant D, Deane D, Campbell I, Thomson J, Jepson C, et al. An immunisation strategy for the protection of cattle against alcelaphine herpesvirus-1-induced malignant catarrhal fever. Vaccine. 2008;26:4461-8.

46. Cunha CW, Knowles DP, Taus NS, O'Toole D, Nicola AV, Aguilar $\mathrm{HC}$, et al. Antibodies to ovine herpesvirus 2 glycoproteins decrease virus infectivity and prevent malignant catarrhal fever in rabbits. Vet Microbiol. 2015;175:349-55.

47. Cunha CW, Taus NS, Dewals BG, Vanderplasschen A, Knowles DP, Li H. Replacement of glycoprotein B in alcelaphine herpesvirus 1 by its ovine herpesvirus 2 homolog: implications in vaccine development for sheep-associated malignant catarrhal fever. mSphere. 2016;1:e0108-16.

Publisher's Note Springer Nature remains neutral with regard to jurisdictional claims in published maps and institutional affiliations. 